\title{
IRIDOIDES, TRITERPENOS E OUTROS CONSTITUINTES DAS CASCAS DO CAULE E FLORES DE Tabebuia caraiba BIGNONIACEAE
}

\author{
Aloízio de O. Soares ${ }^{a}$, Caroline Tieppob ${ }^{\mathrm{b}}$, Luzinátia Ramos Soares , Joaquim Corsino ${ }^{\mathrm{b}}$, Alex Fonseca Souza ${ }^{\mathrm{b}}$, Fernanda R. \\ Garcez $^{\mathrm{b}}$ e Walmir S. Garcez ${ }^{\mathrm{b}, *,(1)}$ \\ ${ }^{a}$ Colégio Militar de Campo Grande, 79115-810 Campo Grande - MS, Brasil \\ 'Instituto de Química, Universidade Federal de Mato Grosso do Sul, 79070-900 Campo Grande - MS, Brasil \\ 'Universidade Estadual de Mato Grosso do Sul, Unidade Universitária de Campo Grande, 79400-000 Campo Grande - MS, Brasil
}

Recebido em 08/08/2019; aceito em 28/01/2020; publicado na web em 06/04/2020

\begin{abstract}
IRIDOIDS, TRITERPENES AND OTHER CONSTITUENTS FROM THE TRUNK BARK AND FLOWERS OF THE Tabebuia caraiba. The trunk bark of Tabebuia caraiba Bignoniaceae was found to contain four novel iridoids, 6-O-E-p-coumaroyljioglutin $\mathrm{D}(2), 6-O$-E-p-coumaroyl-3-demethyl-3-O-ethyljioglutin D (3), 6- $O$ - $E$ - $p$-coumaroyl-1-demethyl-1- $O$-ethyljioglutin D (4), 7-O-E-p-coumaroyljiofuranaldehyde (5), three known iridoids, including one iridoid glucoside, jioglutin D (1), rehmaglutin D (6), 6-O-E-p-coumaroylcatalpol (7). From the flowers were obtained six triterpenes, betulinic acid (8), olean-12-en-3-one (9), $\beta$-amyrin (10), oleanolic acid (11), ursolic acid (12), 3-O-E-p-coumaroylursolic acid (13), $2 \alpha$-hydroxyursolic acid (14), $6 \beta, 19 \alpha$-dihydroxyursolic acid (15), and one phenylethanoid, tyrosol (16), in addition to $\beta$-sitosterol, $\beta$-sitosterol-3- $O$ - $\beta$-D-glucopyranoside and $\beta$-sitosterol-3- $O$ - $\beta$-D-(6'- $O$-acyl)-glucopyranoside and $E$ - $p$-coumaric acid. The structures of the isolated compounds were established on the basis of 1D and 2D NMR spectroscopic techniques. The chemical profile of the trunk bark of the plant was determined by HPLC/DAD/HRESIMS and did not present a good correlation with the phytochemical study. It did not detect most of the isolated substances and allowed to propose the presence of some non-isolated compounds.
\end{abstract}

Keywords: Tabebuia caraiba; Bignoniaceae; iridoids; triterpenes.

\section{INTRODUÇÃO}

A família Bignoniaceae engloba um amplo grupo de plantas (cerca de 800 espécies em 100 gêneros) fisionomicamente muito diverso, encontrando-se desde árvores de grande porte a cipós e arbustos. ${ }^{1}$ Nesse grupo há um número significativo de plantas medicinais, destacando-se os ipês, ${ }^{2}$ e o estudo fitoquímico de bignoniáceas revelou uma grande diversidade de metabólitos secundários, destacando-se as seguintes classes: iridoides, flavonoides, triterpenos, lignanas, quinonas e derivados dos ácidos cinâmico e benzoico. ${ }^{1} \mathrm{O}$ presente trabalho trata do estudo químico das cascas do caule e das flores de Tabebuia caraiba, popularmente conhecida como "paratudo". Foi realizada também uma avaliação do perfil químico das cascas da planta por HPLC/DAD/ MS. A espécie é bastante difundida na medicina popular e seu nome revela a gama de aplicações que lhe são atribuídas, sendo considerada apropriada "para tudo". A casca de T. caraiba, mascada ou ingerida na forma de chá, é indicada popularmente para tratamento de problemas estomacais e do fígado, "amarelão", verminoses, diabetes, febre e malária, além de apresentar propriedades abortivas. ${ }^{2}$ Uma questão a ser destacada sobre esta planta é o fato de que no Pantanal ela ocorre em extensas formações homogêneas, conhecidas como "paratudais", onde são praticamente as únicas espécies arbóreas presentes, enquanto no Cerrado esta espécie ocorre de maneira esparsa, distribuída na vegetação característica deste domínio vegetal. Esse fato leva à dúvida entre os botânicos, se ambas são da mesma espécie ou se a espécie do Pantanal seria outra e, nesse caso, classificada como T. aurea. Das folhas de um espécime de T. caraiba, coletada em Pirassununga - SP, foram caracterizados seis flavonoides: 6-hidroxiluteolina, luteolina-7-O-glucosídeo, quercetina-3-O-glucosídeo, quercetina-3- $O$-galactosídeo, rutina e um diglicosídeo derivado da quercetina (3- $O$-galactose e ramnose), o qual não teve sua estrutura completamente elucidada. ${ }^{3}$

*e-mail: walmir.garcez@ufms.br

\section{PARTE EXPERIMENTAL}

\section{Instrumentação e procedimentos gerais}

Os espectros de $\mathrm{RMN}{ }^{1} \mathrm{H}$ e ${ }^{13} \mathrm{C}$ foram registrados em aparelho Bruker, modelo DPX-300, operando a $300 \mathrm{MHz}\left({ }^{1} \mathrm{H}\right)$ e a $75 \mathrm{MHz}$ $\left({ }^{13} \mathrm{C}\right)$, com amostras dissolvidas em $\mathrm{CDCl}_{3}$, acetona- $\mathrm{d}_{6}$ e TMS como referência interna.

Nas colunas de fracionamento foram utilizados gel de sílica 70-230 e 230-400 mesh (Merck) e Sephadex LH-20 (Pharmacia). Foram utilizadas placas cromatográficas analíticas (CCDA) (2,5 x 7,5 cm, 0,5 g) preparadas com gel de sílica G (Merck) ou cromatofolhas (Merck). Nas separações por cromatografia em camada delgada preparativa (CCDP) foi empregado gel de sílica $60 \mathrm{PF}_{254}$. As revelações das placas cromatográficas foram feitas através de nebulização com solução de sulfato de cério IV a $2 \%$ em $\mathrm{H}_{2} \mathrm{SO}_{4} 2 \mathrm{~N}$ e aquecimento ou pela exposição à luz ultravioleta (366 ou $254 \mathrm{~nm}$ ).

O dados de HPLC/DAD/Massas foram obtidos no cromatógrafo da Shimadzu UFLC LC-20AD/detector diode array acoplado a equipamento de massas ESI qTOF microTOF-Q III (Bruker Daltonics). As condições cromatográficas foram: coluna Kinetex C-18 (2,6 $\mu$, 150x2.2 mm Phenomenex), eluição com gradiente de água e acetonitrila, ambas com $1 \%$ de ácido acético e fluxo de $0,3 \mathrm{~mL} / \mathrm{min}$.

\section{Material vegetal}

O material vegetal foi coletado na reserva de mata nativa existente no campus da Universidade Federal de Mato Grosso do Sul, em Campo Grande - MS (20³0'25.7'S 54³7'01.4”W), em agosto de 2012. A identificação da planta foi realizada pela Prof ${ }^{\mathrm{a}}$. $M s c$. Ubirazilda Maria Resende e uma exsicata, de ${ }^{\circ} 13047$, foi depositada no herbário CGMS/UFMS. O cadastro de acesso foi registrado no SISGEN com o protocolo A1E 3865. 


\section{Extração e isolamento dos constituintes químicos}

As flores in natura $(5,50 \mathrm{~kg})$ foram submetidas à extração por maceração com etanol por sete dias. Após filtragem, a solução resultante foi concentrada sob pressão reduzida, fornecendo o extrato etanólico bruto $(336,35 \mathrm{~g})$. Esse extrato foi dissolvido em $1000 \mathrm{~mL}$ de $\mathrm{MeOH} / \mathrm{H}_{2} \mathrm{O}$ (9:1) e, em seguida, submetido a partições sucessivas com hexano e clorofórmio, obtendo-se assim as fases hexânica (13,53 g), clorofórmica $(6,69$ g) e hidrometanólica.

A fase clorofórmica das flores (C) foi submetida a uma coluna cromatográfica em gel de sílica 70-230 mesh (70 g), eluída com gradiente de hexano/AcOEt/MeOH, obtendo-se 10 frações de $100 \mathrm{~mL}$ cada. Na fração C5 (0,42 g, hexano/AcOEt 1:1) foi identificado o ácido ursólico (12). A fração C6 (1,20 g, AcOEt) foi submetida a coluna cromatográfica em gel de sílica 230-400 mesh, eluída com gradiente hexano/AcOEt/MeOH, obtendo-se 151 frações de $10 \mathrm{~mL}$ cada. A fração C6-61/65 (19,5 mg, Hex/AcOEt//MeOH 70:30:2) tratava-se do ácido $2 \alpha$-hidroxiursólico (14), enquanto na fração C6-145/148 $(18,70 \mathrm{mg}, \mathrm{MeOH})$ foi caracterizado o ácido $E$-p-cumárico. A fração C9 (1,57 g, AcOEt/MeOH 1:1) foi submetida a coluna cromatográfica em gel de sílica 230-400 mesh (57 g), eluída com gradiente hexano/ AcOEt/MeOH, obtendo-se 180 frações de $10 \mathrm{~mL}$ cada. Na fração C9-16/18 (8,4 mg, Hex/AcOEt/MeOH 140:60:15) foi caracterizado o $\beta$-sitosterol-3-O- $\beta$-D-glucopiranosídeo.

As cascas do caule foram secas ao ar, moídas $(2,65 \mathrm{~kg})$ e extraídas por maceração com metanol. A solução resultante após filtração foi concentrada sob pressão reduzida, fornecendo o extrato metanólico bruto (164,02 g). Parte deste extrato metanólico $(64,76 \mathrm{~g})$ foi submetida à cromatografia em coluna de gel de sílica 70-230 mesh (182 g), eluída com gradiente $\mathrm{CHCl}_{3} / \mathrm{MeOH}$, resultando em 30 frações de $150 \mathrm{~mL}$ cada (M). Nas frações M3-5 (120,0 mg) foi identificado o triterpeno olean-12-en-3-ona (9). A fração M8 $(840 \mathrm{mg})$ foi submetida à cromatografia em coluna de Sephadex LH-20 (40 g), eluída isocra-

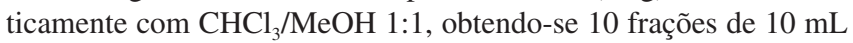
cada. A fração M8-4 (250,0 mg) foi submetida à cromatografia em coluna de gel de sílica 230-400 mesh (40 g), eluída com gradiente $\mathrm{CHCl}_{3} / \mathrm{MeOH}$, resultando em 50 frações de $10 \mathrm{~mL}$ cada. Na fração M8-4-5 (9,4 $\mathrm{mg} \mathrm{CHCl}_{3} / \mathrm{MeOH}$ 99:1) foi identificada a $\beta$-amirina (10), enquanto M8-4-9 (10,7 mg $\left.\mathrm{CHCl}_{3} / \mathrm{MeOH} 99: 1\right)$ foi caracterizada como sendo $\beta$-sitosterol. A fração M11 foi submetida à cromatografia em coluna de gel de sílica 230-400 mesh ( 80 g), eluída com gradiente Hex/AcOEt, obtendo-se 120 frações de $20 \mathrm{~mL}$ cada. As seguintes substâncias foram obtidas deste processo: M11-3 - $\beta$-amirina (10, 15,5 mg, Hex/AcOEt 95:5); M11-13/15 - ácido 3-O-E- $p$-cumaroilursólico (13, 10,0 mg, Hex/AcOEt 90:10); M11-22/25 - ácido betulínico (8, 8,3 mg, Hex/AcOEt 90:10); M11-27 - ácido oleanólico (11, 13,0 mg, Hex/AcOEt 90:10); M11-56/59 - tirosol (16, 22,0 mg, Hex/ AcOEt 70:30); M11-73 - ácido 6 $\beta, 19 \alpha$-di-hidroxiursólico (15, 6,5 mg, Hex/AcOEt 70:30); M11-89/95, após CCDP em CHCl $\mathrm{CH}_{3} / \mathrm{MeOH}$ 95:5 6-O-E-p-cumaroil-3-desmetil-3-O-etiljioglutina D (3) - composto em maior proporção - e 6-O-E-p-cumaroil-1-desmetil-1- $O$-etiljioglutina D (4), em mistura (8,1 mg); M11-100, após cromatografia em coluna de gel de sílica 230-400 mesh, em gradiente de $\mathrm{CHCl}_{3} / \mathrm{MeOH}$, resultou em 60 frações de $10 \mathrm{~mL}$ cada: M11-100-14 - 6-O-E-pcumaroiljioglutina D (2) (8,5 mg, $\left.\mathrm{CHCl}_{3} / \mathrm{MeOH} 98: 2\right)$; M11-100-40 - 7-O-E-p-cumaroiljiofuranaldeído (5) (5,2 mg, $\left.\mathrm{CHCl}_{3} / \mathrm{MeOH} 98: 2\right)$ e M11-100-60: remaglutina D (6, 6,2 mg, $\mathrm{CHCl}_{3} / \mathrm{MeOH}$ 98:2); M11-110/112 - $\beta$-sitosterol-3-O- $\beta$-D-(6'-O-acil)-glucopiranosídeo (10,4 mg, Hex/AcOEt 50:50); M11-117/118 - jioglutina D (1, 5,2 mg, Hex/AcOEt 20:80). A fração M18 (2463,7 mg, $\mathrm{CHCl}_{3} / \mathrm{MeOH}$ 65:35) apresentou-se como um material cristalino, caracterizado como sendo 6-O-E-p-cumaroilcatalpol (7).

6-O-E-p-cumaroilcatalpol (7) Sólido branco. $\mathrm{RMN}{ }^{13} \mathrm{C}\left(\mathrm{CD}_{3} \mathrm{OD}\right.$, $75 \mathrm{MHz}) \delta \mathrm{c}:$ 94,1 (C-1), 141,3 (C-3), 101,9 (C-4), 35,7 (C-5), 80,3 (C-6), 59,2 (C-7), 65,8 (C-8), 42,1 (C-9), 62,0 (C-10), 98,7 (C-1'), 73,9 (C-2'), 77,6 (C-3'), 70,8 (C-4'), 76,8 (C-5'), 60,4 (C-6'), 126,1 (C-1"), 130,3 (C-2" e C-6"), 115,9 (C-3" e C-5"), 160,5 (C-4"), 113,5 (C- $\alpha$ ), 146,2 (C- $\beta$ ), 167,9 (COO).

\section{RESULTADOS E DISCUSSÃO}

O estudo químico das cascas do caule e das flores de Tabebuia caraiba resultou no isolamento e caracterização de sete iridoides (1-7), sendo quatro deles inéditos (2-5), oito triterpenos (8-15) e um feniletanoide (16), além de ácido E-p-cumárico, $\beta$-sitosterol, $\beta$-sitosterol-3- $O$ - $\beta$-D-glucopiranosídeo e $\beta$-sitosterol-3-O- $\beta$-D-(6'-O-acil)-glucopiranosídeo.

A substância 1, cujos espectros de $\mathrm{RMN}{ }^{1} \mathrm{He} \mathrm{e}^{13} \mathrm{C}$ (Tabelas 1 e 2) apresentaram características de iridoides isolados de Bignoniaceae, ${ }^{4}$ foi identificada como sendo jioglutina D (Figura 1). Seu espectro<smiles>[R6]O[C@H]1[C@@H]2C[C@H](OCC)O[C@@H]([2H])[C@H]2[C@]2(CO)O[C@H]12</smiles>

1- $\mathrm{R}_{1}=\mathrm{R}_{2}=\mathrm{OMe}, \mathrm{R}_{3}=\mathrm{H}$

2- $\mathrm{R}_{1}=\mathrm{R}_{2}=\mathrm{OMe}, \mathrm{R}_{3}=E$ - $p$-Cumaroil

3- $\mathrm{R}_{1}=\mathrm{OEt}, \mathrm{R}_{2}=\mathrm{OMe}, \mathrm{R}_{3}=E$ - $p$-Cumaroil

4- $\mathrm{R}_{1}=\mathrm{OMe}, \mathrm{R}_{2}=\mathrm{OEt}, \mathrm{R}_{3}=E$ - $p$-Cumaroil

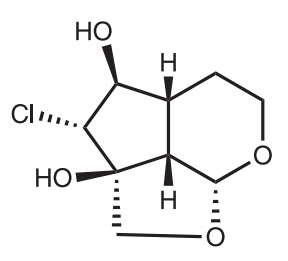

6<smiles>O=CCC1c2cocc2[C@H](OC(=O)/C=C/c2ccc(O)cc2)[C@@H]1O</smiles>

5

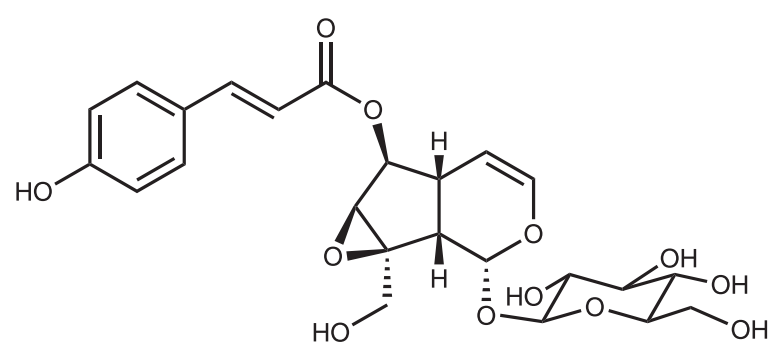

7

Figura 1. Estruturas dos iridoides isolados de Tabebuia caraiba 
de $\mathrm{RMN}{ }^{13} \mathrm{C}$ mostrou 11 sinais, sendo dois relativos a metoxilas, dois a carbonos acetálicos $\left(\delta_{\mathrm{C}} 99,0-\mathrm{C}-1\right.$ e $\left.95,5-\mathrm{C}-3\right)$ e quatro sinais entre $\delta_{\mathrm{C}} 77,7$ e 66,0 , os quais evidenciaram a presença de quatro carbonos oxigenados no anel de cinco membros. Esses dados foram compatíveis com a presença de um grupo hidroxila em C- $6\left(\delta_{\mathrm{C}} 77,7\right)$ e em C-10 $\left(\delta_{\mathrm{C}} 61,2\right)$ e de uma função epóxido em C7-C8 $\left(\delta_{\mathrm{C}} 63,0\right.$ e 66,0, respectivamente). Os dois sinais de $\mathrm{CH}$, em $\delta_{\mathrm{C}} 35,9$ e 40,8 correspondem aos carbonos da junção de anel (C-5 e C-9) e o sinal em $\delta c 29,7\left(\mathrm{CH}_{2}\right)$ a C-4. Esses dados permitiram a identificação de $\mathbf{1}$ como sendo jioglutina $\mathrm{D}$, um iridoide isolado anteriormente apenas de Rehmannia glutinosa (Scrophulariaceae).

$\mathrm{O}$ espectro de $\mathrm{RMN}{ }^{13} \mathrm{C}$ da substância 2 (Tabela 2) mostrou 18 sinais e, entre esses, pôde-se observar um conjunto com deslocamentos químicos muito semelhantes aos observados para o iridoide $\mathbf{1}$. Por outro lado, constatou-se nos espectros de $\mathrm{RMN}{ }^{1} \mathrm{H}$ e de ${ }^{13} \mathrm{C}$ sinais típicos de um grupo E-p-cumaróla ${ }^{6}$ (Tabelas 1 e 2). No espectro de $\mathrm{RMN}{ }^{1} \mathrm{H}$ os deslocamentos químicos de duas metoxilas $\left(\delta_{\mathrm{H}} 3,37 \mathrm{e}\right.$ 3,42 ) confirmaram que nenhuma delas se encontrava ligada ao anel aromático. Desta forma, foi possível propor que a estrutura de $\mathbf{2}$ corresponde à do composto 1 esterificado com ácido $E$ - $p$-cumárico. A localização do grupo $p$-cumaroíla foi definida pela desproteção observada em H-6 $\left(\delta_{\mathrm{H}} 5,20\right)$ em relação ao mesmo hidrogênio em 1 $\left(\delta_{\mathrm{H}} 4,02\right)$ (Tabela 1$)$. A comparação dos valores de ${ }^{13} \mathrm{C}$ destas duas substâncias (Tabela 2) evidenciou também a desproteção de C-6 e a proteção de C-5 e C-7 no composto 2 , variações essas compatíveis com a presença do grupo $p$-cumaroíla em C-6. Portanto, a estrutura de 2 foi definida como 6-O-E-p-cumaroiljioglutina D, um composto inédito na literatura.

As substâncias $\mathbf{3}$ e $\mathbf{4}$ foram obtidas em mistura, numa proporção de 2:1, respectivamente, e se constituíram numa mancha única em cromatografia de camada fina. Os espectros de $\mathrm{RMN}{ }^{1} \mathrm{H}$ e ${ }^{13} \mathrm{C}$ da mistura (Tabelas 1 e 2) revelaram que ambas as substâncias eram iridoides possuindo um resíduo de $E$ - $p$-cumaroíla, destacando-se quatro sinais entre $\delta_{\mathrm{C}} 94,1$ e 98,8 , indicativos de grupo hemi-acetal ou acetal em C-1 e C-3. O conjunto de sinais no espectro de RMN ${ }^{13} \mathrm{C}$ apresentou grande semelhança com os da substância 2 , incluindo os relativos aos carbonos do anel de cinco membros. Comparando-se os dados de $\mathrm{RMN}{ }^{1} \mathrm{H}$ e ${ }^{13} \mathrm{C}$ de $\mathbf{2}, \mathbf{3}$ e $\mathbf{4}$ constatou-se que as principais diferenças encontravam-se nos grupos ligados a $\mathrm{C}-1$ e $\mathrm{C}-3$, as quais indicaram a presença em $\mathbf{3}$ e $\mathbf{4}$ de uma etoxila e uma metoxila em cada estrutura (Tabela 2). As correlações observadas no espectro HMBC permitiram confirmar que na substância 3 há um grupo $\mathrm{OMe}$ em C-1 e OEt em C-3, enquanto na substância 4 ocorre o inverso (OEt em C-1 e OMe em C-3). Assim, a substância 3 foi caracterizada como sendo 6- $O$ - $E$ - $p$-cumaroil-3-desmetil-3- $O$-etiljioglutina $\mathrm{D}$ e a substância 4 como 6-O-E-p-cumaroil-1-desmetil-1- $O$-etiljioglutina $\mathrm{D}$, ambas descritas pela primeira vez na literatura.

No espectro de $\mathrm{RMN}{ }^{13} \mathrm{C}$ da substância 5 (Tabela 2) observaram-se sinais correspondentes a um grupo $E$ - $p$-cumaroíla (Tabela 1 ) e sinais relativos a outros nove carbonos, dos quais quatro são olefínicos e

Tabela 1. Dados de RMN ${ }^{1} \mathrm{H}(300 \mathrm{MHz})$ das substâncias 1-6 - $\delta_{\mathrm{H}}$ (mult.; $J$ em Hz)

\begin{tabular}{|c|c|c|c|c|c|c|}
\hline & $\mathbf{1}^{\mathrm{a}}$ & $2^{\mathrm{b}}$ & $3^{\mathrm{b}}$ & $4^{\mathrm{b}}$ & $\mathbf{5}^{\mathrm{b}}$ & $6^{\mathrm{b}}$ \\
\hline 1 & $4,92(d ; 4,0)$ & $5,00(d ; 3,7)$ & $4,98(d ; 3,8)$ & $5,10(d ; 3,8)$ & $7,20(s)$ & $5,25(d ; 5,5)$ \\
\hline $3 \mathrm{a}$ & $4,68(d d ; 6,6 ; 2,7)$ & $4,74(d d ; 6,0 ; 2,6)$ & $4,85(m)$ & $4,79(m)$ & $9,95(t ; 1,2)$ & $\begin{array}{c}3,47(d d d d ; 11,7 ; 5,7 \\
1,5 ; 0,8)\end{array}$ \\
\hline $3 b$ & - & - & - & - & - & $\begin{array}{c}3,81(d d d ; 13,3 ; 11,7 \\
2,9)\end{array}$ \\
\hline $4 a$ & $1,75(m)$ & $1,79(d t ; 13,7 ; 7,7)$ & $1,70-1,95(\mathrm{c})$ & & 3,09 (ddd; 17,3; 4,5; 1,2) & $1,67(m)$ \\
\hline $4 b$ & $1,83(\mathrm{~m})$ & $1,89(d d d ; 13,7 ; 5,3 ; 2,7)$ & & & $2,75(d d d ; 17,3 ; 9,2 ; 1,2)$ & $1,78(m)$ \\
\hline 5 & $1,92(m)$ & $2,19(m)$ & $2,20(m)$ & & $3,42(m)$ & $2,17(m)$ \\
\hline 6 & $4,02(d ; 7,9)$ & $5,20(d d ; 8,4 ; 0,8)$ & $5,12(d d ; 9,8 ; 1,3)$ & $5,20(d d ; 9,8 ; 1,3)$ & $4,26(d d ; 8,50 ; 5,2)$ & $3,89(d d ; 10,3 ; 9,9)$ \\
\hline 7 & $3,24(s)$ & $3,54(d ; 0,8)$ & $3,50(s l)$ & & $5,85(d ; 5,2)$ & $4,14(d d ; 10,3 ; 1,6)$ \\
\hline 8 & - & - & - & - & - & - \\
\hline 9 & $2,61(d d ; 7,9 ; 4,0)$ & $2,75(d d ; 8,2 ; 3,7)$ & $2,75(d d ; 8,2 ; 3,8)$ & & - & $2,29(d d ; 10,3 ; 5,5)$ \\
\hline $10 \mathrm{a}$ & $3,47(d ; 12,4)$ & $3,52(d ; 12,7)$ & $3,80(\mathrm{c})$ & & $7,50(s)$ & $3,41(d d ; 10,3 ; 1,6)$ \\
\hline $10 \mathrm{~b}$ & $3,95(d ; 12,4)$ & $4,02(d ; 12,7)$ & & & & $4,33(d ; 10,3)$ \\
\hline 1-OMe & $3,35(s)$ & $3,35(s)$ & & & & \\
\hline 3-OMe & $3,36(s)$ & $3,40(s)$ & & & & \\
\hline \multicolumn{7}{|l|}{ COO- } \\
\hline$\alpha$ & & $6,36(d ; 16,0)$ & $6,36(d ; 16,0)$ & & $6,35(d ; 16,0)$ & \\
\hline$\beta$ & & $7,62(d ; 16,0)$ & $7,67(d ; 16,0)$ & & $7,65(d ; 16,0)$ & \\
\hline \multicolumn{7}{|l|}{$1^{\prime}$} \\
\hline 2'e 6' & & $7,55(d ; 8,6)$ & $7,54(d ; 8,6)$ & & $7,54(d ; 8,60)$ & \\
\hline 3'e 5' & & $6,87(d ; 8,6)$ & $6,87(d ; 8,6)$ & & $6,89(d ; 8,60)$ & \\
\hline \multicolumn{7}{|l|}{$4^{\prime}$} \\
\hline $\mathrm{O}_{\mathbf{C H}} \mathrm{CH}_{3}$ & & & $3,77-3,89(\mathrm{c})$ & $4,08-3,95(\mathrm{c})$ & & \\
\hline $\mathrm{OCH}_{2} \mathbf{C H}_{3}$ & & & $1,13(t ; 7,0)$ & $1,20(t ; 7,0)$ & & \\
\hline $\mathrm{OCH}_{3}$ & & & $3,41(s)$ & $3,50(s)$ & & \\
\hline
\end{tabular}

${ }^{\mathrm{a}} \mathrm{CDCl}_{3}$; ${ }^{\mathrm{b}}$ Acetona- $\mathrm{d}_{6}$; ${ }^{\mathrm{c}}$ sobrepostos. 
Tabela 2. Dados de RMN ${ }^{13} \mathrm{C}$ (75 MHz) das substâncias 1-6

\begin{tabular}{|c|c|c|c|c|c|c|}
\hline & $\mathbf{1}^{\mathrm{a}}$ & $2^{\mathrm{b}}$ & $3^{\mathrm{b}}$ & $4^{\mathrm{b}}$ & $5^{\text {b }}$ & $6^{\mathrm{b}}$ \\
\hline 1 & 99,0 & 98,7 & 98,8 & 97,1 & 135,9 & 100,8 \\
\hline 3 & 95,5 & 95,3 & 94,1 & 95,7 & 202,1 & 55,8 \\
\hline 4 & 29,7 & 29,9 & 30,5 & 30,5 & 47,0 & 21,8 \\
\hline 5 & 35,9 & 33,4 & 34,1 & 34,1 & 38,2 & 36,5 \\
\hline 6 & 77,7 & 80,1 & 80,5 & 80,7 & 83,4 & 75,9 \\
\hline 7 & 63,0 & 60,5 & 61,1 & 61,1 & 69,6 & 74,9 \\
\hline 8 & 66,0 & 66,7 & 66,7 & 68,3 & 131,0 & 84,9 \\
\hline 9 & 40,8 & 40,8 & 41,3 & 41,2 & 131,2 & 45,7 \\
\hline 10 & 61,2 & 61,0 & 64,0 & 63,3 & 138,5 & 72,4 \\
\hline 1-OMe & 55,3 & 55,0 & - & - & - & - \\
\hline 3-OMe & 54,7 & 55,5 & - & - & - & - \\
\hline COO- & - & 167,8 & 167,2 & 167,8 & 167,0 & - \\
\hline$\alpha$ & - & 115,2 & 115,2 & 115,2 & 116,7 & - \\
\hline$\beta$ & - & 145,9 & 145,4 & 145,9 & 145,8 & - \\
\hline 1 ' & - & 133,7 & 133,1 & 133,7 & 127,0 & - \\
\hline 2'e 6' & - & 131,1 & 131,5 & 131,1 & 133,6 & - \\
\hline 3' е 5 ' & - & 116,7 & 116,1 & 116,7 & 116,7 & - \\
\hline $4^{\prime}$ & - & 160,7 & 160,4 & 160,7 & 160,6 & - \\
\hline $\mathrm{OCH}_{2} \mathrm{CH}_{3}$ & - & - & 61,5 & 61,5 & - & - \\
\hline $\mathrm{OCH}_{2} \mathrm{CH}_{3}$ & - & - & 15,6 & 15,9 & - & - \\
\hline $\mathrm{OCH}_{3}$ & - & - & 55,0 & 56,1 & - & - \\
\hline
\end{tabular}

${ }^{\mathrm{a}} \mathrm{CDCl}_{3}$; bacetona- $\mathrm{d}_{6}$.

dois oxigenados. Os sinais em $\delta_{\mathrm{C}} 202,1$ e $\delta_{\mathrm{H}} 9,95(1 \mathrm{H}, t, J=1,2 \mathrm{~Hz})$ indicaram a presença de um grupo carbonila de aldeído, sendo que o padrão de acoplamento do hidrogênio, juntamente com o obervado no experimento $\operatorname{COSY}{ }^{1} \mathrm{H}^{-}{ }^{1} \mathrm{H}$, revelou a conectividade com um grupo $\mathrm{CH}_{2}\left(\delta_{\mathrm{C}} 47,0\right)$. Esse experimento mostrou, também, a conexão do $\mathrm{CH}_{2}$ com um grupo $\mathrm{CH}\left(\delta_{\mathrm{H}} 3,42 ; \delta_{\mathrm{C}} 38,2\right)$. Os quatro sinais no espectro de $\mathrm{RMN}{ }^{13} \mathrm{C}$ referentes aos carbonos olefínicos em $\delta_{\mathrm{C}} 131,0,131,2$, 135,9 e 138,5 , juntamente com os dois singletos em $\delta_{\mathrm{H}} 7,20$ e 7,50, no espectro de $\mathrm{RMN}{ }^{1} \mathrm{H}$, e suas respectivas correlações observadas no espectro HMQC, indicaram a existência de um anel furano 3,4-dissubstituído na estrutura de $\mathbf{5}$.

A associação dos dados dos experimentos HMQC, HMBC e $\mathrm{COSY}{ }^{1} \mathrm{H}-{ }^{1} \mathrm{H}$ permitiu definir a conectividade do grupo $\mathrm{CH}-\mathrm{CH}_{2}-\mathrm{CHO}$ com os dois carbonos oxigenados $\mathrm{CH}$, numa sequência.

A associação desta estrutura parcial com a do furano levou à proposta da estrutura mostrada na Figura 1. A localização do grupo cumaroíla em C-7 foi definida com base no acoplamento observado no experimento HMBC entre H-7 e o carbono carboxílico. Um composto com esse esqueleto carbônico, denominado Jiofuraldeído dimetilacetal, foi obtido dos frutos de Catalpa sp., Bignoniaceae. ${ }^{7}$ Assim, a substância 5 foi caracterizada como sendo 7-O-E-pcumaroiljiofuranaldeído e é inédita na literatura.

A substância 6, assim como as anteriores, apresentou características de iridoides em seus espectros de $\mathrm{RMN}{ }^{1} \mathrm{He}{ }^{13} \mathrm{C}$, exibindo 9 sinais neste último, porém com apenas um carbono acetálico $\left(\delta_{\mathrm{C}} 100,8\right)$. Sendo assim, esse sinal foi atribuído a $\mathrm{C}-1$ enquanto que o relativo a um carbono metilênico em $\delta_{\mathrm{C}} 55,8$ foi associado a C-3 e os sinais em $\delta_{\mathrm{C}} 21,8,36,5$ e 45,7 a C-4, C-5 e C-9, respectivamente. Para os outros três carbonos do anel ciclopentânico e C-10 corresponderam os sinais em $\delta_{\mathrm{C}} 84,9$ (C,C-8), 75,9 (CH,C-6), 74,9 (CH,C-7) e 72,4 $\left(\mathrm{CH}_{2}, \mathrm{C}-10\right)$. Estes valores sugeriram que os carbonos correspondentes encontravam-se ligados a hidroxilas. Um iridoide contendo essas características já havia sido descrito como produto natural, ${ }^{8}$ porém, não se observou uma boa correlação entre os dados de $\mathrm{RMN}{ }^{13} \mathrm{C}$ das duas substâncias. No entanto, os dados espectrais de $\mathbf{6}$ foram perfeitamente correlacionados com os de remaglutina $\mathrm{D}$, um iridoide clorado em C-7 e possuindo um anel oxetânico entre C-10 e C-1. ${ }^{8}$ Os valores de $J$ também se mostraram compatíveis, indicando que a estereoquímica das substâncias é coincidente, como, por exemplo, as constantes de acoplamento entre H-7 e H-6 $(J=10,3 \mathrm{~Hz})$ e entre H-6 e H-5 $(J=9,9 \mathrm{~Hz})$, demonstrando a relação trans entre eles. Assim, a substância 6 foi identificada como sendo remaglutina D, que, embora possuindo uma estrutura incomum, já foi obtida de algumas plantas medicinais chinesas. ${ }^{5,8}$ No entanto, esse é o primeiro relato da ocorrência deste iridoide clorado em uma planta brasileira e também na família Bignoniaceae.

A substância majoritária encontrada nas cascas do caule de T. caraiba foi caracterizada como sendo o iridoide 6-O-E-pcumaroilcatalpol ou especiosídeo (7). O espectro de $\mathrm{RMN}{ }^{13} \mathrm{C}$ desta substância apresentou, além dos sinais típicos de um grupo $E$ - $p$ cumaroíla e de uma unidade $\beta$-D-glucosila, nove sinais atribuíveis a um iridoide contendo uma ligação dupla C-3/C-4 $\left(\delta_{C} 141,3\right.$ e 101,9) e um carbono acetálico em C-1 $\left(\delta_{\mathrm{C}} 94,1\right)$. Os sinais restantes no espectro de $\mathrm{RMN}{ }^{13} \mathrm{C}$, em conjunto com os presentes no espectro de $\mathrm{RMN}{ }^{1} \mathrm{H}$, levaram à estrutura correspondente à do 6-O-E-p-cumaroilcatalpol, iridoide já isolado de uma outra espécie de Tabebuia (T. rosea), ${ }^{9}$ cujos dados espectrais mostraram-se bastante semelhantes aos de 7 . A identificação desta substância foi também confirmada através de comparação com amostra autêntica disponível no laboratório.

Dentre os oito triterpenos pentacíclicos isolados, quatro são de ampla distribuição no reino vegetal e foram identificados através da análise dos dados espectrais e comparação com os relatados na literatura como ácido betulínico (8), olean-12-en-3-ona (9), $\beta$-amirina (10), ácido oleanólico (11), ácido ursólico (12) ${ }^{10}$ Os demais, de ocorrência menos comum em plantas, foram caracterizados como sendo ácido 3-O-E-p-cumaroil-ursólico (13), ${ }^{11}$ ácido 2 $\alpha$-hidroxiursólico (14) e ácido $6 \beta, 19 \alpha$-di-hidroxiursólico, conhecido também como ácido uncárico (15), e estão sendo descritos pela primeira vez no gênero Tabebuia, com exceção de $\mathbf{1 5}$, que foi obtido de $T$. rosea.${ }^{12} \mathrm{O}$ composto 16 foi identificado como o feniletanoide tirosol [2-(4-hidroxifenil)-etanol], com base em seus dados de $\mathrm{RMN}{ }^{1} \mathrm{H}$ e ${ }^{13} \mathrm{C}$ e por comparação com os mesmos descritos na literatura. ${ }^{13} \mathrm{O}$ tirosol é mencionado como inibidor do crescimento de Candida albicans, ${ }^{14}$ destacando-se também por apresentar atividade antifúnfica in vitro sobre Aspergillus niger e Fusarium oxysporium. ${ }^{15}$

As estruturas dos demais compostos isolados, ácido E-pcumárico, $\beta$-sitosterol, $\beta$-sitosterol-3-O- $\beta$-D-glucopiranosídeo e $\beta$-sitosterol-3-O- $\beta$-D-(6'-O-acil)-glucopiranosídeo foram determinadas com base nos respectivos dados espectrais e por comparação com amostras autênticas.

\section{Perfil químico}

Foram obtidos os cromatogramas de íons totais (HPLC/DAD/ HRESIMS) do extrato da casca de $T$. caraiba nos modos positivo e negativo. Através do programa "Compass DataAnalysis 4.1 Bruker ${ }^{\circledR}$ ” foi possível determinar as prováveis fórmulas moleculares (Tabela 3), considerando erros de massa e "mSigma" abaixo de 5 ppm e 30, respectivamente. Os dados dos espectros de massas - $\mathrm{MS}^{2}$ dos íons e de UV foram comparados com as informações relatadas na literatura, visando uma maior confiabilidade na identificação dos compostos. A busca na literatura foi realizada utilizando a plataforma on-line "Scifinder", tendo como filtro os trabalhos relacionados com espécies do gênero Tabebuia. A análise desses dados revelou que, 
Tabela 3. Substâncias identificadas por HPLC/DAD/MS na casca do caule de Tabebuia caraiba

\begin{tabular}{|c|c|c|c|c|c|c|c|}
\hline Pico & Compostos (CAS) & $\mathrm{UV}_{(\mathrm{nm})}$ & RT & {$[\mathrm{M}+\mathrm{H}]_{\mathrm{m} / \mathrm{z}}^{+}$} & {$[\mathrm{M}-\mathrm{H}]^{-} \mathrm{m} / \mathrm{z}$} & $\mathrm{FM}^{\mathrm{b}}$ & $\mathrm{MS} / \mathrm{MS}_{\text {(modo negativo) }}$ \\
\hline 17 & 4-hidroximetil-2-metoxifenil- hexosil-diglicosídeo & 284 & 8.0 & 449,1643 & 447,1489 & $\mathrm{C}_{19} \mathrm{H}_{28} \mathrm{O}_{12}$ & 191 \\
\hline 18 & 2-(4-hidroxifenil)etil- hexosil-diglicosídeo & 278 & 10.2 & 433,1704 & 431,1522 & $\mathrm{C}_{19} \mathrm{H}_{28} \mathrm{O}_{11}$ & 191 \\
\hline 19 & 3,4,5-trimetoxifenil- hexosil-diglicosídeo & 284 & 12.3 & 479,1760 & 477,1564 & $\mathrm{C}_{20} \mathrm{H}_{30} \mathrm{O}_{13}$ & - \\
\hline 20 & Estereospermosídeo (879090-40-1) & 294,309 & 13.1 & 527,1782 & 525,1569 & $\mathrm{C}_{24} \mathrm{H}_{30} \mathrm{O}_{13}$ & $205 ; 163$ \\
\hline 21 & Ácido E-p-cumárico a (7400-08-0) & 296,309 & 13.7 & 165,0549 & 163,0379 & $\mathrm{C}_{9} \mathrm{H}_{8} \mathrm{O}_{3}$ & - \\
\hline 22 & Verminosídeo (50932-19-9) & 292,329 & 18.3 & 525,1607 & 523,1451 & $\mathrm{C}_{24} \mathrm{H}_{28} \mathrm{O}_{13}$ & $247 ; 203 ; 179 ; 161$ \\
\hline 23 & 6-O-Cumaroil-harpagídeo (1423129-05-8) & 296,309 & 19.3 & 511,1811 & 509,1605 & $\mathrm{C}_{24} \mathrm{H}_{30} \mathrm{O}_{12}$ & $347 ; 329 ; 163 ; 145$ \\
\hline 7 & 6-O-E-Cumaroilcatalpol ou Especiosídeo a (72514-90-0) & 298,313 & 20.9 & 509,1657 & 507,1503 & $\mathrm{C}_{24} \mathrm{H}_{28} \mathrm{O}_{12}$ & $\begin{array}{c}328 ; 231 ; 203 ; 163 ; \\
145\end{array}$ \\
\hline 24 & 6-O-E-Feruloilcatalpol (770721-33-0) & 296,312 & 22.0 & 539,1741 & 537,1593 & $\mathrm{C}_{25} \mathrm{H}_{30} \mathrm{O}_{13}$ & $193 ; 175 ; 160$ \\
\hline
\end{tabular}

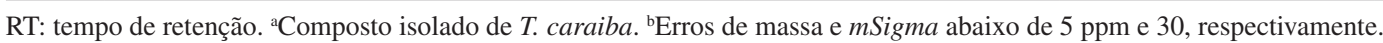

das substâncias isoladas, apenas ácido cumárico (5), esse obtido das flores, e 6-O-E-p-cumaroilcatalpol (7) foram detectados, sendo que o iridoide 7 se constitui no pico base do cromatograma. No entanto, algumas substâncias não isoladas no estudo fitoquímico foram detectadas: três aril-hexosil-diglicosídeos e quatro iridoides (Tabela 3). A presença dessas substâncias é coerente com o conjunto de substâncias isoladas e possivelmente não foram obtidas no estudo fitoquímico realizado por se encontrarem em baixa concentração. O estudo realizado por Santos et al. ${ }^{16}$ utilizando HPLC/DAD/MS identificou 13 substâncias nas cascas de T. aurea, que pode ser considerada uma sinonímia de $T$. caraiba. Dentre essas, apenas duas substâncias foram isoladas no presente estudo fitoquímico da casca do caule: 6-O-E- $p$ cumaroilcatalpol (7) e ácido cumárico (5). No estudo de Santos et al. foi proposta a identificação de seis iridoides, dos quais três foram caracterizados no perfil químico relatado no presente artigo.

\section{CONCLUSÃO}

Foi realizada a investigação fitoquímica da casca do caule e das flores e de um espécime da planta medicinal Tabebuia caraiba, conhecida como "paratudo", e analisado o perfil químico das cascas da planta. O espécime estudado foi coletado em região de Cerrado, tendo sido isoladas e caracterizadas 21 substâncias, incluindo sete iridoides e oito triterpenos. Desses, quatro iridoides são inéditos (2-5) e dois iridoides (1 e 6) e quatro triterpenos $(\mathbf{8}, \mathbf{1 2}-\mathbf{1 4})$ são descritos pela primeira vez no gênero. A análise do perfil químico da casca do caule mostrou que, das sete substâncias isoladas desta mesma parte da planta, apenas uma foi detectada por HPLC/DAD/HRESIMS, enquanto sete substâncias não isoladas, possivelmente componentes minoritárias, foram detectadas. Estes resultados evidenciam que o estudo fitoquímico ainda se constitui numa ferramenta fundamental na investigação da composição química das plantas, pois permite fornecer informacões seguras sobre os constituintes químicos majoritários presentes (e muitas vezes, também os minoritários), que nem sempre podem ser detectados pela técnica supracitada.

\section{MATERIAL SUPLEMENTAR}

Espectros de RMN de ${ }^{1} \mathrm{He}^{13} \mathrm{C}$ das substâncias isoladas das cascas do caule e flores de Tabebuia caraiba e os dados de HPLC/DAD/ MS estão disponíveis gratuitamente em http://quimicanova.sbq.org. br, na forma de arquivo PDF.

\section{AGRADECIMENTOS}

À FUNDECT-MS, ao CNPq, à CAPES e à CPq-PROPP/UFMS pelas bolsas e/ou auxílios concedidos e à $M S c$. U. M. Resende (INBIO/UFMS) pela identificação do material vegetal.

\section{REFERÊNCIAS}

1. Oliveira, A. B.; Raslan, D. S.; Miaraglia, M. C. M.; Mesquita, A. A. L.; Quim. Nova 1990, 13, 302.

2. Pott, A.; Pott, V. J.; Plantas do Pantanal, EMBRAPA: Corumbá, 1994.

3. Blatt, C. T. T.; Salatino, A.; Salatino, M. L. F.; Biochem. Syst. Ecol. 1996, 24, 89.

4. Schripsema, J.; Henriques, A. T.; Jensen, S. R.; Biochem. Syst. Ecol. 2000, 28, 351 .

5. Morota, T.; Sasaki, H.; Sugama, K.; Nishimura, H.; Chin, M.; Mitsuhashi, H.; Phytochemistry 1990, 29, 523.

6. Lu, Y.; Foo L. Y.; Phytochemistry 2000, 263, 55.

7. Machida, K.; Ikeda, C.; Kakuda, R.; Yaoita, Y.; Kikuchi, M.; Nat. Med. 2001, 55, 61 .

8. Morota, T.; Nishimura, H.; Sasaki, H.; Chin, M.; Sugama, K.; Katsuhara, K.; Mitsuhashi, H.; Phytochemistry 1989, 28, 2385.

9. Compadre, C. M.; Jauregui, J. F.; Nathan, P. J.; Enríquez, R. G.; Planta Med. 1982, 46, 42.

10. Ahmad, U. V.; Rahman, A.; Handbook of Natural Products Data. Pentacyclic Triterpenoids, Volume 2, Elsevier: Amsterdam, 1994.

11. David, J. P.; Meira, M.; David, J. M.; Guedes, M. L. da S.; Quim. Nova 2004, 27, 62 .

12. Oliveira, M. E.; Lemos, T. L. G.; Braz-Filho, R.; Rev. Bras. Farm. 1999, $80,46$.

13. Pouchert, C.; Behnke, J.; The Aldrich Library of ${ }^{13} \mathrm{C}$ and ${ }^{I} \mathrm{H}$ FT NMR Spectra, Volume 2, Aldrich Chemical Company Inc.: Milwaukee, 1993.

14. Chen, H.; Li, S.; Zhou, Z.; Bai, N.; Ho, C.; ACS Symp. Ser. 2006, 925, 195.

15. Rocha, A. D.; Braga, F. C.; Oliveira, A. B.; Lombardi, J. A.; de Souza Filho, J. D.; Phytother. Res. 2004, 18, 463.

16. Dos Santos, V. S.; Macedo, F. A.; Do Vale, J. S.; Silva, D. B.; Carollo, C. A.; Metabolomics 2017, 13, 72. 